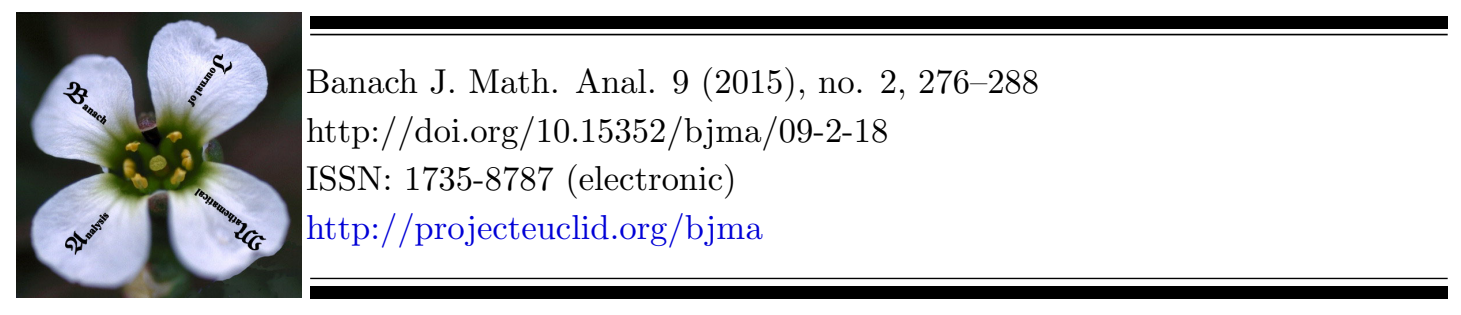

\title{
LOCALLY PSEUDOCONVEX INDUCTIVE LIMIT OF SEQUENCES OF LOCALLY PSEUDOCONVEX ALGEBRAS
}

\author{
MATI ABEL ${ }^{1}$ AND REYNA MARÍA PÉREZ-TISCAREÑO ${ }^{2 *}$ \\ Communicated by M. Joiţa
}

\begin{abstract}
Conditions such that a locally $k$-convex inductive limit of a sequence of $k_{n}$-normed algebras is a locally $m$-( $k$-convex) algebra, are given. It is shown that every locally pseudoconvex inductive limit $E$ of a sequence of commutative locally $m$-pseudoconvex algebras is a commutative locally $m$-pseudoconvex algebra if the multiplication in $E$ is jointly continuous.
\end{abstract}

\section{INTRODUCTION}

A. Arosio asked in [4, p. 349] whether any locally convex inductive limit of normed algebras is a locally $m$-convex algebra. An answer to this question has been given in [3, p. 114] (see also [11, Theorem 15.4]), by showing that every locally convex inductive limit of a countable family of normed algebras is a locally $m$-convex algebra (another proof for this fact was given in [6, Theorem 1]). In this paper we give an analogous result in case of locally $k$-convex inductive limit of $k_{n}$-normed algebras. Moreover, it is shown that a locally pseudoconvex inductive limit $E$ of commutative locally $m$-pseudoconvex algebras is a topological algebra of the same type as the factors if the multiplication in $E$ is jointly continuous. In the locally convex case a similar result has been proved in [7].

Date: Received: Mar. 16, 2014; Revised: Jun. 16, 2014; Accepted: Aug. 28, 2014.

* Corresponding author.

2010 Mathematics Subject Classification. Primary 46H05; Secondary 46H20.

Key words and phrases. Topological algebra, locally $m$-pseudoconvex algebra, locally $m$-( $k$-convex $)$ algebra, $k$-normed algebra, locally pseudoconvex inductive limit of topological algebras. 


\section{Preliminaries}

Let $E$ be a unital topological algebra over $\mathbb{K}$, the field of real numbers $\mathbb{R}$ or complex numbers $\mathbb{C}$, with separately continuous multiplication (in short, a topological algebra). If the underlying topological linear space of $E$ is locally pseudoconvex (see [12, p. 4] or [13, p. 4]), then $E$ is called a locally pseudoconvex algebra. In this case, $E$ has a base $\mathcal{U}=\left\{U_{\lambda}: \lambda \in \Lambda\right\}$ of neighborhoods of zero consisting of balanced $\left(\mu U_{\lambda} \subset U_{\lambda}\right.$ when $\left.|\mu| \leqslant 1\right)$ and pseudoconvex $\left(U_{\lambda}+U_{\lambda} \subset \mu U_{\lambda}\right.$ for $\mu \geqslant 2)$ sets. This base defines a set of numbers $\left\{k_{\lambda}: \lambda \in \Lambda\right\}$ in $(0,1]$ (see, for instance, [13, pp. 3-6] or [9, pp. 161-162]) such that

$$
U_{\lambda}+U_{\lambda} \subset 2^{\frac{1}{k_{\lambda}}} U_{\lambda}
$$

and

$$
\Gamma_{k_{\lambda}}\left(U_{\lambda}\right) \subset 2^{\frac{1}{k_{\lambda}}} U_{\lambda} \text { for each } \lambda \in \Lambda
$$

where

$$
\Gamma_{k}(U)=\left\{\sum_{\nu=1}^{n} \mu_{\nu} u_{\nu}: n \in \mathbb{N}, u_{1}, \cdots, u_{n} \in U, \mu_{1}, \cdots, \mu_{n} \in \mathbb{K} \text { with } \sum_{\nu=1}^{n}\left|\mu_{\nu}\right|^{k} \leqslant 1\right\}
$$

for any subset $U$ of $E$ and $k \in(0,1]$. The set $\Gamma_{k}(U)$ is the absolutely $k$-convex hull of $U$ in $E$. A subset $U \subset E$ is called absolutely $k$-convex if $U=\Gamma_{k}(U)$ and absolutely pseudoconvex if $U=\Gamma_{k}(U)$ for some $k \in(0,1]$. In case when

$$
\inf \left\{k_{\lambda}: \lambda \in \Lambda\right\}=k>0,
$$

$E$ is a locally $k$-convex algebra and when $k=1$, then $E$ is a locally convex algebra. A locally m-pseudoconvex (multiplicative pseudoconvex) algebra is a topological algebra which has a base of neighborhoods of zero which consist of $m$-pseudoconvex (that is, idempotent and absolutely pseudoconvex) sets. A locally $m$-( $k$-convex) algebra is a topological algebra which has a base of neighborhoods of zero, which are $m$-( $k$-convex) (that is, idempotent and absolutely $k$-convex). In case when $k=1, E$ is a locally $m$-convex algebra.

The topology on a locally pseudoconvex algebra $E$ can be defined by a family $\mathcal{P}=\left\{p_{\lambda}: \lambda \in \Lambda\right\}$ of $k_{\lambda}$-homogeneous seminorms $p_{\lambda}$ (that is, $p_{\lambda}(\mu a)=|\mu|^{k_{\lambda}} p_{\lambda}(a)$ for each $\mu \in \mathbb{K}$ and $a \in E$ ), defined by the base neighborhood $U_{\lambda}$ of zero, where $k_{\lambda} \in(0,1]$ is the power of nonhomogeneity of $p_{\lambda}$ for each $\lambda \in \Lambda$ and $p_{\lambda}$ has been defined by

$$
p_{\lambda}(a)=\inf \left\{|\mu|^{k_{\lambda}}: a \in \mu \Gamma_{k_{\lambda}}\left(U_{\lambda}\right)\right\}
$$

for each $a \in E$ and $\lambda \in \Lambda$ (see [13, pp. 3-6], [5, pp. 189 and 195] or [1, pp. 15-16]).

When the topology an of algebra $E$ is defined by a $k$-homogeneous submultiplicative norm $\|\cdot\|$ for some $k \in(0,1]$, then $E$ is called a $k$-normed algebra and $\|e\|=1$ whenever $E$ has a unit $e$. 
Let $\left(E_{n}\right)_{n \in \mathbb{N}}$ be a sequence of locally pseudoconvex algebras and for every $m, n \in \mathbb{N}$ with $m \leqslant n$ let

$$
f_{n m}: E_{m} \rightarrow E_{n}
$$

be a homomorphism such that

1) $f_{n n}=i d_{E_{n}}$ for every $n \in \mathbb{N}$ and

2) $f_{o n}=f_{o m} \circ f_{m n}$ for any $m, n, o \in \mathbb{N}$ such that $n \leqslant m \leqslant o$.

The sequence of locally pseudoconvex algebras $\left(E_{n}\right)_{n \in \mathbb{N}}$ with the maps $f_{n m}$ defined above is called an inductive system of locally pseudoconvex algebras and it is denoted by $\left(E_{n}, f_{m n}\right)$.

Let $E_{0}$ be the disjoint union of algebras $E_{\alpha}$. That is,

$$
E_{0}=\bigcup_{n \in \mathbb{N}}\left\{(a, n): a \in E_{n}\right\} \text {. }
$$

Then, $x, y \in E_{0}$ (that is, $x=\left(x_{0}, n\right)$ with $x_{0} \in E_{n}$ and $y=\left(y_{0}, m\right)$ with $y_{0} \in E_{m}$ for some $n$ and $m$ in $\mathbb{N}$ ) are equivalent (in short $x \sim y$ ) if there exists $o \in \mathbb{N}$ such that $n \leqslant o, m \leqslant o$ and

$$
f_{\text {on }}\left(x_{0}\right)=f_{\text {om }}\left(y_{0}\right) \text {. }
$$

The quotient set $E_{0} / \sim$ is called the inductive (or direct) limit of the inductive system $\left(E_{n}, f_{m n}\right)$. We shall denote this by $\lim \left(E_{n}, f_{m n}\right)$ or simply by $\lim E_{n}$.

For every $n \in \mathbb{N}$, let $i_{n}: E_{n} \rightarrow E_{0}$ be the canonical injection or natural injection (that is, $i_{n}(x)=(x, n)$ for each $\left.x \in E_{n}\right)$ and $\pi: E_{0} \rightarrow E_{0} / \sim$ the quotient map. Then,

$$
f_{n}=\pi \circ i_{n}: E_{n} \rightarrow E=\lim _{\longrightarrow} E_{n} \text { for every } n \in \mathbb{N}
$$

is the canonical map from $E_{n}$ to $E$.

We endow $E_{0}$ with the disjoint union topology (that is, with the topology

$$
\left\{U \subset E_{0}: i_{n}^{-1}(U) \in \tau_{n} \text { for every } n \in \mathbb{N}\right\},
$$

where $\tau_{n}$ denotes the topology of $E_{n}$. Here $i_{n}$ is an open and closed continuous map. When all algebras $E_{n}$ are subalgebras of some algebra $E$, then every $i_{n}$ is an inclusion $E_{n} \rightarrow E$. In this case, we endow $E_{0}$ with the coherent topology

$$
\left.\left\{U \subset E_{0}: U \cap E_{n} \in \tau_{n} \text { for every } n \in \mathbb{N}\right\}\right)
$$

and the inductive limit $E$ we endow with the final topology $\tau_{\lim E_{n}}$ (the inductive limit topology), defined by the homomorphisms $f_{n}$ (that is

$$
\left.\tau_{\underline{\lim } E_{n}}=\left\{U \subset E: f_{n}^{-1}(U) \in \tau_{n} \text { for every } n \in \mathbb{N}\right\}\right) .
$$

A base of neighborhoods of zero in this topology is

$$
\left\{O \subset E: O \text { is balanced and } f_{n}^{-1}(O) \in \mathcal{N}_{n} \text { for every } \mathrm{n} \in \mathbb{N}\right\},
$$

(in particular, when every $E_{n}$ is a subalgebra of $E$, then

$$
\left\{O \subset E: O \text { is balanced and } O \cap E_{n} \in \mathcal{N}_{n} \text { for every } n \in \mathbb{N}\right\} \text { ), }
$$


where $\mathcal{N}_{n}$ denotes the set of all neighborhoods of zero in $E_{n}$. Then, $f_{n}$ is a continuous (open) map for every $n \in \mathbb{N}$. Since

$$
E=\bigcup_{n \in \mathbb{N}} f_{n}\left(E_{n}\right)
$$

and $f_{m} \circ f_{m n}=f_{n}$ when $n \leqslant m$ (because $\left.i_{n}\left(x_{n}\right) \sim i_{m}\left(f_{m n}\left(x_{n}\right)\right)\right)$ we get $f_{n}\left(E_{n}\right) \subseteq$ $f_{m}\left(E_{m}\right)$ for any $m, n \in \mathbb{N}$ with $n \leqslant m$.

The algebraic operations in $\lim E_{n}$ are defined as usual (see [10, p. 110]): for every $x, y \in E$ (then $x \in f_{n}\left(E_{n}\right)$ and $y \in f_{m}\left(E_{m}\right)$ for some $m, n \in \mathbb{N}$ ) there exists $o \in \mathbb{N}$ such that $m \leqslant o, n \leqslant o, x=f_{o}\left(x_{o}\right)$ and $y=f_{o}\left(y_{o}\right)$ for some $x_{o}, y_{o} \in E_{o}$. So, the algebraic operations in $E$ are defined by

$$
x+y=f_{o}\left(x_{o}+y_{o}\right), \quad \lambda x=f_{o}\left(\lambda x_{o}\right), \quad x y=f_{o}\left(x_{o} y_{o}\right)
$$

for every $\lambda \in \mathbb{K}$. With respect to such algebraic operations, $\left(E, \tau_{\lim E_{n}}\right)$ is a topological algebra (see [10, p. 115]).

Since the topology $\tau_{\lim E_{n}}$ on $E$ is not necessarily locally pseudoconvex, we consider on $E$ the final locally pseudoconvex topology $\tau$ (see [2, pp. 1952-1953]) defined by the base of neighborhoods at $x \in E_{n}$ in the form

$$
\mathcal{L}_{x}=\left\{x+U: U \text { is absolutely pseudoconvex in } E \text { and } f_{n}^{-1}(U) \in \mathcal{N}_{n}\right\}
$$

where $\mathcal{N}_{n}$ denotes again the set of all neighborhoods of zero in $E_{n}$. Similarly as in [10, pp. 115-116], it is easy to show that $(E, \tau)$ is a locally pseudoconvex algebra.

In this paper, we consider inductive limits of sequences $\left(E_{n}\right)_{n \in \mathbb{N}}$ of locally pseudoconvex algebras such that $E_{n}$ is a subalgebra of $E_{n+1}$ with continuous inclusion and the locally pseudoconvex inductive limit topology $\tau$ induces a topology coarser than the initial topology of $E_{n}$ for each $n \in \mathbb{N}$.

\section{On LOCAlly $k$-CONVEX IndUCtive Limit of A SEQUENCE OF LOCALly $k_{n}$-CONVEX ALGEBRAS}

It was shown in [4, Proposition 12] that any commutative locally convex inductive limit $E$ of a countable family of normed algebras is locally $m$-convex. Later on, in [3, Theorem 2.1], it was shown that the commutativity of $E$ in this result can be omitted (another proof of this fact has been given in [6, Theorem 1]). To show a similar result in the case when $E$ is a locally pseudoconvex inductive limit of a sequence of $k_{n}$-normed algebras $\left(E_{n},\|\cdot\|_{n}\right)$ with $k_{n} \in(0,1]$ for each $n \in \mathbb{N}$, we need the next.

Lemma 3.1. Let $B, C$ be two subsets of an algebra and $k \in(0,1]$. Then, $\Gamma_{k}(B) \Gamma_{k}(C) \subset \Gamma_{k}(B C)$. In particular, if $U$ is an idempotent set, then $\Gamma_{k}(U)$ is also idempotent.

Proof. Take $x \in \Gamma_{k}(B)$ and $y \in \Gamma_{k}(C)$. Then,

$$
x=\sum_{n=1}^{p} a_{n} x_{n} \quad \text { and } \quad y=\sum_{m=1}^{q} b_{m} y_{m},
$$


where $x_{1}, \ldots, x_{p} \in B, y_{1}, \ldots, y_{q} \in C$,

$$
\sum_{n=1}^{p}\left|a_{n}\right|^{k} \leq 1 \quad \text { and } \quad \sum_{m=1}^{q}\left|b_{m}\right|^{k} \leq 1
$$

Hence

$$
x y=\left(\sum_{n=1}^{p} a_{n} x_{n}\right)\left(\sum_{m=1}^{q} b_{m} y_{m}\right)=\sum_{n=1}^{p} \sum_{m=1}^{q} a_{n} b_{m} x_{n} y_{m},
$$

where $x_{n} y_{m} \in B C$ and

$$
\sum_{n=1}^{p} \sum_{m=1}^{q}\left|a_{n} b_{m}\right|^{k}=\sum_{n=1}^{p} \sum_{m=1}^{q}\left|a_{n}\right|^{k}\left|b_{m}\right|^{k}=\left(\sum_{n=1}^{p}\left|a_{n}\right|^{k}\right)\left(\sum_{m=1}^{q}\left|b_{m}\right|^{k}\right) \leq 1 .
$$

Theorem 3.2. Let $(E, \tau)$ be a locally $k$-convex inductive limit of a sequence of $k_{n}$-normed algebras $\left(E_{n},\|\cdot\|_{n}\right)$ with continuous inclusions. If $k, k_{n} \in(0,1]$ and $k \leqslant k_{n}$ for each $n \in \mathbb{N}$, then $(E, \tau)$ is a locally $m$-( $k$-convex) algebra.

Proof. For any $n \in \mathbb{N}$, let $B_{n}=\left\{x \in E_{n}:\|x\|_{n} \leqslant 1\right\}$ (the unit ball in $E_{n}$ ), and let $k \in(0,1]$ be a number such that $k \leqslant k_{n}$ for each $n \in \mathbb{N}$. Then, $B_{n}$ is an idempotent and absolutely $k$-convex set for each $n \in \mathbb{N}$. Indeed, if $a, b \in B_{n}$ and

$$
|\lambda|^{k}+|\mu|^{k} \leqslant 1
$$

then

$$
\|\lambda a+\mu b\|_{n} \leqslant|\lambda|^{k_{n}}\|a\|_{n}+|\mu|^{k_{n}}\|b\|_{n} \leqslant|\lambda|^{k_{n}}+|\mu|^{k_{n}} \leqslant|\lambda|^{k}+|\mu|^{k} \leqslant 1 .
$$

Hence, $\lambda a+\mu b \in B_{n}$. Taking this into account, we can assume that every norm $\|\cdot\|_{n}$ is $k$-homogeneous otherwise, instead of $\|\cdot\|_{n}$, we consider the new norm

$$
\|\cdot\|_{n}^{\frac{k}{k_{n}}}
$$

which is $k$-homogeneous.

Moreover, we can assume that $B_{n-1} \subseteq B_{n}$ for each $n>1$. Otherwise, we replace $k$-norm $\|\cdot\|_{n}$ of the algebra $E_{n}$ with equivalent $k$-norm $\|\cdot\|_{n}^{\prime}$ such that $B_{n-1}^{\prime} \subseteq B_{n}^{\prime}$ for each $n>1$ where $B_{n}^{\prime}=\left\{a \in E_{n}:\|a\|_{n}^{\prime} \leqslant 1\right\}$. Because the injection $E_{n-1} \rightarrow E_{n}$ is a continuous linear map, there exists $M_{n} \geqslant 1$ such that $\|a\|_{n} \leqslant M_{n}\|a\|_{n-1}$ for each $a \in E_{n-1}$ (see [5, Proposition 4.3.11], both norms here are $k$-homogeneous). We consider first the case when $E_{n-1}$ and $E_{n}$ have the same unit element $e_{n}$. Let $\|a\|_{1}^{\prime}=\|a\|_{1}$ (then $\|a\|_{2} \leqslant M_{2}^{\prime}\|a\|_{1}^{\prime}$ where $M_{2}^{\prime}=M_{2}$ ) and

$$
\|a\|_{2}^{\prime}=\sup _{c \in E_{2}, q_{2}(c) \leqslant 1} q_{2}(a c)
$$

where

$$
q_{2}(a)=\sup _{s \in B_{1}^{\prime}}\|s a\|_{2}
$$

for all $a \in E_{2}$. Then

$$
\begin{gathered}
q_{2}(\lambda a)=|\lambda|^{k} q_{2}(a), \quad q_{2}(a+b) \leqslant q_{2}(a)+q_{2}(b), \\
\|a\|_{2} \leqslant q_{2}(a) \leqslant \sup _{s \in B_{1}^{\prime}}\|s\|_{2}\|a\|_{2} \leqslant M_{2}^{\prime}\|a\|_{2}
\end{gathered}
$$


and

$$
q_{2}(a b)=\sup _{s \in B_{1}^{\prime}}\|s(a b)\|_{2} \leqslant \sup _{s \in B_{1}^{\prime}}\|s a\|_{2}\|b\|_{2}=q_{2}(a)\|b\|_{2} \leqslant q_{2}(a) q_{2}(b)
$$

for each $\lambda \in \mathbb{K}$ and $a, b \in E_{2}$. Hence, $q_{2}$ is a $k$-norm on $E_{2}$ which is equivalent to $\|\cdot\|_{2}$. Taking this into account, $\|\cdot\|_{2}^{\prime}$ is a $k$-homogeneous norm on $E_{2}$. Moreover,

$$
\|a b\|_{2}^{\prime}=\left(\left\|l_{a b}\right\|_{2}\right)_{o p}=\left(\left\|l_{a} \circ l_{b}\right\|_{2}\right)_{o p} \leqslant\left(\left\|l_{a}\right\|_{2}\right)_{o p}\left(\left\|l_{b}\right\|_{2}\right)_{o p}=\|a\|_{2}^{\prime}\|b\|_{2}^{\prime}
$$

(here $\|a\|_{2}^{\prime}$ is the operator norm $\left(\left\|l_{a}\right\|_{2}\right)_{o p}$ of the left regular representation $l_{a}$ of $a$ on $\left.\left(E_{2}, q_{2}\right)\right)$,

$$
q_{2}(a)=q_{2}\left(a e_{2}\right)=M_{2}^{\prime} q_{2}\left(a \frac{e_{2}}{M_{2}^{\prime \frac{1}{k}}}\right) \leqslant M_{2}^{\prime} \sup _{c \in E_{2}, q_{2}(c) \leqslant 1} q_{2}(a c)=M_{2}^{\prime}\|a\|_{2}^{\prime}
$$

because $q_{2}\left(e_{2}\right) \leqslant M_{2}^{\prime}$ and

$$
\frac{1}{M_{2}^{\prime}}\|a\|_{2} \leqslant \frac{1}{M_{2}^{\prime}} q_{2}(a) \leqslant\|a\|_{2}^{\prime} \leqslant \sup _{c \in E_{2}, q_{2}(c) \leqslant 1} q_{2}(a) q_{2}\left(e_{2} c\right)=q_{2}(a)\left\|e_{2}\right\|_{2}^{\prime} \leqslant M_{2}^{\prime}\|a\|_{2}
$$

for each $a, b \in E_{2}$. Since

$$
\|t\|_{2}^{\prime}=\sup _{c \in E_{2}, q_{2}(c) \leqslant 1} q_{2}(t c)=\sup _{c \in E_{2}, q_{2}(c) \leqslant 1} \sup _{s \in B_{1}^{\prime}}\|(s t) c\|_{2} \leqslant \sup _{c \in E_{2}, q_{2}(c) \leqslant 1} q_{2}(c)=1
$$

for each $t \in B_{1}^{\prime}$ (because $B_{1}^{\prime}=B_{1}$ and $B_{1} t \subset B_{1}$ ), then $\|\cdot\|_{2}^{\prime}$ is a $k$-norm on $E_{2}$, which is equivalent to $\|\cdot\|_{2}$, and satisfies the condition $B_{1}^{\prime} \subseteq B_{2}^{\prime}$.

The norm $\|\cdot\|_{3}^{\prime}$ we define similarly, that is, we put

$$
\|a\|_{3}^{\prime}=\sup _{c \in E_{3}, q_{3}(c) \leqslant 1} q_{3}(a c)
$$

where

$$
q_{3}(a)=\sup _{s \in B_{2}^{\prime}}\|s a\|_{3}
$$

for all $a \in E_{3}$. Now, similarly as above, we have $\|a\|_{3} \leqslant M_{3}^{\prime}\|a\|_{2}^{\prime}$ for $M_{3}^{\prime}=M_{3} M_{2}$, $\frac{1}{M_{3}^{\prime}}\|a\|_{3} \leqslant\|a\|_{3}^{\prime} \leqslant M_{3}^{\prime}\|a\|_{3}$ for each $a \in E_{3}$ and $\|a\|_{3}^{\prime} \leqslant 1$ for each $a \in B_{2}^{\prime}$. Hence, $B_{2}^{\prime} \subseteq B_{3}^{\prime}$. Continuing in the same way, for every fixed $n \geqslant 4$ we define

$$
\|a\|_{n}^{\prime}=\sup _{c \in E_{n}, q_{n}(c) \leqslant 1} q_{n}(a c)
$$

where

$$
q_{n}(a)=\sup _{s \in B_{n-1}^{\prime}}\|s a\|_{n}
$$

for all $a \in E_{n}$ and show that $B_{n-1}^{\prime} \subseteq B_{n}^{\prime}$.

Let now $E_{n-1}$ and $E_{n}$ be arbitrary $k$-normed algebras. Instead of these algebras, we consider direct products $E_{n-1} \times \mathbb{K}$ and $E_{n} \times \mathbb{K}$ which are $k$-normed algebras with respect to the algebraic operations (similarly as in case of the unitization) and norm $\|(a, \lambda)\|_{k}=\|a\|_{k}+|\lambda|$ for each $(a, \lambda) \in E_{k} \times \mathbb{K}$ (here $k$ is $n-1$ or $n)$. Then $E_{n-1} \times \mathbb{K}$ and $E_{n} \times \mathbb{K}$ have the same unit element $(\theta, 1)$, where $\theta$ is the zero element in $E_{n-1}$ and $E_{n}$. Moreover, $E_{n-1} \times \mathbb{K}$ is a subalgebra of $E_{n} \times \mathbb{K}$. Hence, there are equivalent $k$-norms $\|(\cdot, \cdot)\|_{n-1}^{\prime}$ and $\|(\cdot, \cdot)\|_{n}^{\prime}$ such that $\|(a, \lambda)\|_{n}^{\prime} \leqslant\|(a, \lambda)\|_{n-1}^{\prime}$ if $\|(a, \lambda)\|_{n-1}^{\prime} \leqslant 1$. Thus

$$
\|a\|_{n}^{\prime}=\|(a, 0)\|_{n}^{\prime} \leqslant\|(a, 0)\|_{n-1}^{\prime}=\|a\|_{n-1}^{\prime}
$$


for each $a \in B_{n-1}^{\prime}$. Hence, $B_{n-1}^{\prime} \subseteq B_{n}^{\prime}$ for each fixed $n>1$. Consequently, we can assume that

$$
B_{1} \subseteq B_{2} \subseteq \cdots \subseteq B_{n} \subseteq \cdots
$$

Since $B_{1}$ is bounded in $B_{2}$ (because $\|a\|_{2} \leqslant M_{2}\|a\|_{1}$ for each $a \in E_{1}$ ) and $B_{2}$ is a neighborhood of zero in $E_{2}$, then there is a number $t_{1} \geqslant 1$ such that $B_{1} \subset t_{1} B_{2}$. We put $B_{1}^{\prime}=B_{1}$ and

$$
B_{n}^{\prime}=\Gamma_{k}\left(I\left(B_{n-1} \bigcup \frac{1}{t_{n-1}} B_{n}\right)\right)=\Gamma_{k}\left(\bigcup_{j \in \mathbb{N}}\left(B_{n-1} \bigcup \frac{1}{t_{n-1}} B_{n}\right)^{j}\right)
$$

for $n>1$, where $I(U)$ is the idempotent hull (see [8, pp. 26 and 27]) of $U \subset E$. Then, $B_{2}^{\prime}$ is an idempotent (by Lemma 3.1) and absolutely $k$-convex set. Because

$$
B_{1} \cup \frac{1}{t_{1}} B_{2} \subset B_{2} \subset B_{2} \cup \frac{1}{t_{2}} B_{3},
$$

then $B_{2}^{\prime} \subset B_{3}^{\prime}$ (it is clear that $I(U) \subset I(V)$ and $\Gamma_{k}(U) \subset \Gamma_{k}(V)$ if $\left.U \subset V\right)$. Since

$$
\frac{1}{t_{1}} B_{2} \subset\left(B_{1} \cup \frac{1}{t_{1}} B_{2}\right) \subseteq I\left(B_{1} \cup \frac{1}{t_{1}} B_{2}\right) \subseteq \Gamma_{k}\left(I\left(B_{1} \cup \frac{1}{t_{1}} B_{2}\right)\right)=B_{2}^{\prime},
$$

then, continuing in the same way, we have an increasing sequence $\left\{B_{n}^{\prime}: n \geqslant 2\right\}$ of idempotent and absolutely $k$-convex sets $B_{n}^{\prime}$ such that

$$
\frac{1}{t_{n-1}} B_{n} \subset B_{n}^{\prime}
$$

Moreover, $B_{2}^{\prime} \subset t_{1} B_{2}$. Indeed, for $x \in \frac{1}{t_{1}} \bigcup_{j \in \mathbb{N}}\left(B_{1} \cup \frac{1}{t_{1}} B_{2}\right)^{j}$ we have $t_{1} x \in\left(B_{1} \cup \frac{1}{t_{1}} B_{2}\right)^{j_{0}}$ for some $j_{0} \in \mathbb{N}$. Hence there is an element $y \in B_{1} \cup \frac{1}{t_{1}} B_{2}$ such that $t_{1} x=y^{j_{0}}$. If $y \in B_{1}$, then from $t_{1} x \in B_{1}^{j_{0}} \subset B_{1} \subset t_{1} B_{2}$ follows that $x \in B_{2}$, otherwise $y \in \frac{1}{t_{1}} B_{2}$. Then, from $t_{1} x \in \frac{1}{t_{1}^{j_{0}}} B_{2}^{j_{0}} \subset \frac{1}{t_{1}^{j_{0}}} B_{2}$ follows that $x \in \frac{1}{t_{1}^{j_{0}+1}} B_{2} \subset B_{2}$ provided that $B_{2}$ is balanced. Arguing similarly, we have

$$
B_{n}^{\prime} \subset t_{n-1} B_{n}
$$

where $t_{n} \geqslant 1$ for each $n \in \mathbb{N}$. Thus,

$$
\frac{1}{t_{n-1}} B_{n} \subset B_{n}^{\prime} \subset t_{n-1} B_{n}
$$

for all $n \in \mathbb{N}$.

Now, we shall prove that

$$
\mathcal{L}_{\theta}=\left\{\Gamma_{k}\left(\bigcup_{n \in \mathbb{N}} \varepsilon_{n} B_{n}^{\prime}\right): \varepsilon_{n} \in(0,1]\right\}
$$

is a base of neighborhoods of zero in $E$ which consists of idempotent absolutely $k$-convex sets. Clearly, every element of $\mathcal{L}_{\theta}$ is absolutely $k$-convex, to prove that every element $V=\Gamma_{k}\left(\bigcup_{n \in \mathbb{N}} \varepsilon_{n} B_{n}^{\prime}\right)$ in $\mathcal{L}_{\theta}$ is idempotent, we consider 
$x, y \in \bigcup_{n \in \mathbb{N}} \varepsilon_{n} B_{n}^{\prime}$. Then, $x \in \varepsilon_{n} B_{n}^{\prime}$ and $y \in \varepsilon_{m}^{\prime} B_{m}^{\prime}$ for some $m, n \in \mathbb{N}$. If $B_{n}^{\prime} \subseteq B_{m}^{\prime}$ (the case $B_{n}^{\prime} \supset B_{m}^{\prime}$ is similar), then

$$
x y \in \varepsilon_{n} B_{m}^{\prime} \varepsilon_{m}^{\prime} B_{m}^{\prime} \subseteq \varepsilon_{n} \varepsilon_{m}^{\prime} B_{m}^{\prime} B_{m}^{\prime} \subseteq \varepsilon_{n} B_{m}^{\prime} \subset \bigcup_{n \in \mathbb{N}} \varepsilon_{n} B_{n}^{\prime},
$$

that is, $\bigcup_{n \in \mathbb{N}} \varepsilon_{n} B_{n}^{\prime}$ is idempotent and hence $V$ is idempotent by Lemma 3.1.

To show that $\mathcal{L}_{\theta}$ is a base of neighborhoods of zero for some topology $\tau^{\prime}$ on $E$, we show that $\mathcal{L}_{\theta}$ satisfies the following conditions:

1) if $V \in \mathcal{L}_{\theta}$, then the zero element $\theta \in V$;

2) if $V_{1}, V_{2} \in \mathcal{L}_{\theta}$, then there exists a set $V_{3} \in \mathcal{L}_{\theta}$ such that $V_{3} \subset V_{1} \cap V_{2}$;

3) if $V \in \mathcal{L}_{\theta}$, then there exists a set $V_{0} \in \mathcal{L}_{\theta}$ and for every $y \in V_{0}$ a set $W=y+V_{0}$ such that $W \subset V$.

Clearly 1) holds. To show that 2) holds, we put $V_{1}=\Gamma_{k}\left(\bigcup_{n \in \mathbb{N}} \varepsilon_{n} B_{n}^{\prime}\right)$, $V_{2}=\Gamma_{k}\left(\bigcup_{n \in \mathbb{N}} \varepsilon_{n}^{\prime} B_{n}^{\prime}\right)$ and $\varepsilon_{n}^{\prime \prime}=\inf \left\{\varepsilon_{n}, \varepsilon_{n}^{\prime}\right\}$ for every $n \in \mathbb{N}$. Since $\frac{\varepsilon_{n}^{\prime \prime}}{\varepsilon_{n}} \leqslant 1$ and $\frac{\varepsilon_{n}^{\prime \prime}}{\varepsilon_{n}^{\prime}} \leqslant 1$, then

$$
\varepsilon_{n}^{\prime \prime} B_{n}^{\prime} \subseteq \varepsilon_{n} B_{n}^{\prime}, \quad \varepsilon_{n}^{\prime \prime} B_{n}^{\prime} \subseteq \varepsilon_{n}^{\prime} B_{n}^{\prime}
$$

and hence

$$
\varepsilon_{n}^{\prime \prime} B_{n}^{\prime} \subset\left(\bigcup_{n \in \mathbb{N}} \varepsilon_{n} B_{n}^{\prime}\right) \cap\left(\bigcup_{n \in \mathbb{N}} \varepsilon_{n}^{\prime} B_{n}^{\prime}\right) \subset V_{1} \cap V_{2}
$$

for every $n \in \mathbb{N}$. Thus, we can put

$$
V_{3}=\Gamma_{k}\left(\bigcup_{n \in \mathbb{N}} \varepsilon_{n}^{\prime \prime} B_{n}^{\prime}\right)
$$

Then,

$$
V_{3} \subset \Gamma_{k}\left(V_{1} \cap V_{2}\right) \subset \Gamma_{k}\left(V_{1}\right) \cap \Gamma_{k}\left(V_{2}\right)=V_{1} \cap V_{2} .
$$

3) If $V \in \mathcal{L}_{\theta}$, then

$$
V=\Gamma_{k}\left(\bigcup_{n \in \mathbb{N}} \varepsilon_{n} B_{n}^{\prime}\right)
$$

for some sequence $\left(\varepsilon_{n}\right)$, where $\varepsilon_{n} \in(0,1]$ for each $n \in \mathbb{N}$. Since $V$ is $k$-convex, $2^{-\frac{1}{k}} V+2^{-\frac{1}{k}} V \subset V$. Moreover, $2^{-\frac{1}{k}} V \in \mathcal{L}_{\theta}$ since $2^{-\frac{1}{k}} V=\Gamma_{k}\left(\bigcup_{n \in \mathbb{N}} 2^{-\frac{1}{k}} \varepsilon_{n} B_{n}^{\prime}\right)$, where $2^{-\frac{1}{k}} \varepsilon_{n} \in(0,1]$ for every $n \in \mathbb{N}$. Thus $V_{0}=2^{-\frac{1}{k}} V \in \mathcal{L}_{\theta}$ and $W=y+V_{0} \subset V$ for every $y \in V_{0}$. Consequently, by Theorem 4.5 from [14], $\mathcal{L}_{\theta}$ is a base of neighborhoods of zero for a locally $m$-(k-convex) topology $\tau^{\prime}$ on E.

Claim that $\tau=\tau^{\prime}$. For it, let $O$ be a neighborhood of zero in the topology $\tau^{\prime}$. Then, there exists a neighborhood $U$ of zero such that

$$
U=\Gamma_{k}\left(\bigcup_{n \in \mathbb{N}} \varepsilon_{n} B_{n}^{\prime}\right)
$$

for some sequence $\left(\varepsilon_{n}\right)$, where $\varepsilon_{n} \in(0,1]$ for each $n \in \mathbb{N}$, and $U \subseteq O$. Take $n_{0} \in \mathbb{N}$ and let $f_{n_{0}}: E_{n_{0}} \rightarrow E$ be the canonical map ( $f_{n_{0}}$ is the inclusion). Since 
$\frac{1}{t_{n_{0}-1}} B_{n_{0}} \subset B_{n_{0}}^{\prime}$ by $(2)$, then

$$
f_{n_{0}}^{-1}(U)=\Gamma_{k}\left(\bigcup_{n \in \mathbb{N}} \varepsilon_{n} B_{n}^{\prime}\right) \cap E_{n_{0}} \supset \varepsilon_{n_{0}} B_{n_{0}}^{\prime} \supset \frac{\varepsilon_{n_{0}}}{t_{n_{0}-1}} B_{n_{0}},
$$

where $\frac{\varepsilon_{n_{0}}}{t_{n_{0}-1}} B_{n_{0}}$ is a neighborhood of zero in $E_{n_{0}}$. Thus, $f_{n}^{-1}(U)$ is a neighborhood of zero in $E_{n}$ for every $n \in \mathbb{N}$. Hence, by (1), $U$ is a neighborhood of zero in $E$ in the topology $\tau$. Thus $\tau^{\prime} \subseteq \tau$.

To prove that $\tau \subseteq \tau^{\prime}$, let $U$ be a neighborhood of zero in the topology $\tau$. Then, there is in $E$ an absolutely $k$-convex neighborhood $V$ of zero such that $V \subset U$ and $f_{n}^{-1}(V)=V \cap E_{n}$ is a neighborhood of zero in $E_{n}$ for every $n \in \mathbb{N}$. Since $\left\{\varepsilon_{n} B_{n}: \varepsilon_{n}>0\right\}$ is a base of neighborhoods of zero in $\left(E_{n}, \tau_{n}\right)$ (see [12, p. 14]), then $\varepsilon_{n} B_{n} \subset E_{n} \cap V \subset V$ for some $\varepsilon_{n}<1$. As it has been shown in (3), $B_{n}^{\prime} \subset t_{n-1} B_{n}$ with $t_{n-1} \geqslant 1$. Therefore $\frac{\varepsilon_{n}}{t_{n-1}} B_{n}^{\prime} \subset V$, where $\frac{\varepsilon_{n}}{t_{n-1}} \in(0,1]$ for every $n$. Hence, from

$$
\bigcup_{n \in \mathbb{N}} \frac{\varepsilon_{n}}{t_{n-1}} B_{n}^{\prime} \subset V
$$

it follows

$$
\Gamma_{k}\left(\bigcup_{n \in \mathbb{N}} \frac{\varepsilon_{n}}{t_{n-1}} B_{n}^{\prime}\right) \subset \Gamma_{k}(V)=V \subset U .
$$

Hence, $\tau \subseteq \tau^{\prime}$. It means that $\tau=\tau^{\prime}$.

Corollary 3.3. Locally $k$-convex inductive limit of a sequence of locally $k$-normed algebras with continuous inclusions is a locally $m$-(k-convex) algebra for every $k \in(0,1]$.

\section{LOCALLY PSEUDOCONVEX INDUCTIVE LIMIT OF LOCALLY $m$-PSEUDOCONVEX ALGEBRAS}

It is known that the inductive limit of locally $m$-convex algebras is not necessarily a locally $m$-convex algebra (see the example in [6]). It was shown in [7, Theorem, p. 150] that the locally convex inductive limit $E$ of a sequence of commutative locally $m$-convex algebras is a locally $m$-convex algebra if the multiplication in $E$ is jointly continuous. Next we prove an analogous result for the case of locally pseudoconvex inductive limit of a sequence of commutative locally $m$-pseudoconvex algebras.

Theorem 4.1. Let $E$ be a locally pseudoconvex inductive limit of a sequence of commutative locally $m$-pseudoconvex algebras $E_{n}$ with continuous inclusions. If the multiplication is jointly continuous in $E$, then $E$ is a commutative locally m-pseudoconvex algebra.

Proof. Let $U$ be a neighborhood of zero in $E$. Then, there is a neighborhood $V_{1} \subset U$ of zero such that $\Gamma_{k}\left(V_{1}\right)=V_{1}$ for some $k \in(0,1]$. By the jointly continuity of multiplication in $E$, there exists a neighborhood $O_{1}$ of zero such that $O_{1} O_{1} \subset V_{1}$. Now we put $V_{2}=O_{1} \cap V_{1}$. Then, by the jointly continuity 
of multiplication, there exists a neighborhood $\mathrm{O}_{2}$ of zero such that $\mathrm{O}_{2} \mathrm{O}_{2} \subset \mathrm{V}_{2}$. Inductively we define $V_{v+1}=O_{v} \cap V_{v}$ for each $v \geqslant 1$. Since,

$$
V_{1} \supset V_{2} \supset \ldots \supset V_{v} \supset \ldots,
$$

then $V_{v} \subset U$ for every $v \in \mathbb{N}$.

Since the canonical map (the inclusion) $f_{n}: E_{n} \rightarrow E$ is continuous for every $n \in \mathbb{N}$, there exists for every $v \in \mathbb{N}$ an $m$-pseudoconvex neighborhood $V_{n, v}$ of zero in $E_{n}$ such that $V_{n, v} \subset V_{v}$. Now, for every $n \in \mathbb{N}$, we put $V_{n, 1}^{\prime}=V_{n, 1}$ and

$$
V_{n, v+1}^{\prime}=V_{n, v}^{\prime} \cap V_{n, v+1}
$$

for $v \geqslant 1$. Then,

$$
V_{n, v+1}^{\prime} \subset V_{n, v}^{\prime} \text { for all } n, v \in \mathbb{N}
$$

and $\left(V_{n, v}^{\prime}\right)$ is a sequence of idempotent neighborhoods of zero in $E_{n}$ (since $V_{n, v}$ is an idempotent neighborhood of zero in $E_{n}$ ) for all $n, v \in \mathbb{N}$.

Let $n_{0} \in \mathbb{N}$ and $1 \leqslant p<n_{0}$ be fixed. We define a new sequence $\left(V_{n, v}^{\prime \prime}\right)$ of idempotent neighborhoods of zero in $E_{n}$ as follows: we put $V_{p, 1}^{\prime \prime}=V_{p, 1}$ and for $v \geqslant 1$ put

$$
V_{p, v+1}^{\prime \prime}=V_{n_{0}, v+1}^{\prime} \cap V_{p, v}^{\prime \prime}
$$

and

$$
V_{n, v}^{\prime \prime}=V_{n, v}^{\prime} \text { for } n \geqslant n_{0} \text { and } v \in \mathbb{N} .
$$

So, by definition of $\left(V_{n, v}^{\prime \prime}\right),(4),(5)$ and $(6)$, we have that

$$
V_{n, v+1}^{\prime \prime} \subseteq V_{n, v}^{\prime \prime} \text { for all } v, n \in \mathbb{N}
$$

and from

$$
V_{n_{0}, s}^{\prime \prime} V_{p, q}^{\prime \prime} \subset V_{n_{0}, s}^{\prime \prime} V_{p, s}^{\prime \prime} \subset V_{n_{0}, s}^{\prime} V_{n_{0}, s}^{\prime} \subset V_{n_{0}, s}^{\prime} \subset V_{n_{0}, s} \subset V_{s} \subset V_{1}
$$

it follows that

$$
V_{n_{0}, s}^{\prime \prime} V_{p, q}^{\prime \prime} \subset V_{n_{0}, s}^{\prime \prime} \subset V_{1}
$$

for every natural number $p$ with $p \leqslant n_{0}$ and every natural numbers $s$ and $q$ with $s \leqslant q$.

For any numbers $v(1), \ldots, v(r) \in \mathbb{N}$ with $1=v(0)<v(1)<v(2)<\ldots<v(r)$ and $n(1), \ldots, n(r+1) \in \mathbb{N}$ (arbitrary $r+1$ (not necessarily different and ordered) numbers) we show by induction on $r \in \mathbb{N}$ that

$$
V_{n(1), 1}^{\prime \prime} V_{n(2), v(1)}^{\prime \prime} \cdots V_{n(r+1), v(r)}^{\prime \prime} \subset V_{1}
$$

For $r=1$, (9) holds by (8) (if $n(2)>n(1)$, we can rename these numbers).

Now, we suppose that (9) is true for $r-1$ and prove that (9) is true for $r$ too. Again, we can assume that $n(r) \geq n(r+1$ ) (otherwise we can rename the numbers). Then, using also (8), we get

$$
\begin{gathered}
\left(V_{n(1), 1}^{\prime \prime} V_{n(2), v(1)}^{\prime \prime} \cdots V_{n(r-1), v(r-2)}^{\prime \prime}\right) V_{n(r), v(r-1)}^{\prime \prime} V_{n(r+1), v(r)}^{\prime \prime} \subset \\
\left(V_{n(1), 1}^{\prime \prime} V_{n(2), v(1)}^{\prime \prime} \cdots V_{n(r-1), v(r-2)}^{\prime \prime}\right) V_{n(r), v(r-1)}^{\prime \prime}
\end{gathered}
$$

and by the induction hypothesis, we get the assertion. 
Now, we put $W_{n}=V_{n, n}^{\prime \prime}$. Then, using (7)

$$
W_{v(r)}=V_{v(r), v(r)}^{\prime \prime} \subset V_{v(r), v(r)-1}^{\prime \prime} \subset V_{v(r), v(r-1)}^{\prime \prime}
$$

for each $r \in \mathbb{N}$. Therefore

$$
W_{v(1)} W_{v(2)} \cdots W_{v(r)} \subset V_{v(1), 1}^{\prime \prime} V_{v(2), v(1)}^{\prime \prime} \cdots V_{v(r), v(r-1)}^{\prime \prime} \subset V_{1}
$$

by (9) (which holds for any choice of $r+1$ natural numbers $n(1), \ldots, n(r)$ and $n(r+1))$.

Take $m(1), \ldots, m(s) \in \mathbb{N}$ (arbitrary fixed not necessarily different $s$ natural numbers). We can find $r \leq s$ natural numbers $v(1), \ldots v(r)$ such that

$$
1<v(1)<v(2)<\ldots<v(r)
$$

and the set

$$
\{m(1), \ldots, m(s)\}=\{v(1), \ldots, v(r)\} .
$$

By commutativity of $E_{n}$ and idempotency of $W_{n}$, we have

$$
W_{m(1)} \cdots W_{m(s)}=\prod_{i=1}^{r} W_{v(i)}^{|j: m(j)=v(i)|} \subset \prod_{i=1}^{r} W_{v(i)} \subset V_{1}
$$

for every $r \in \mathbb{N}$, see also (10). Put

$$
W:=\bigcup_{s \in \mathbb{N}}\left(\bigcup_{(m(1), \ldots, m(s)) \in \mathbb{N}^{s}} W_{m(1)} \cdots W_{m(s)}\right) .
$$

Then, $W$ is an idempotent subset of $V_{1}$. Indeed, if $x, y \in W$, then

$$
x \in \bigcup W_{m(1)} \cdots W_{m\left(s_{0}\right)},
$$

where the union is taken over all $\left(m(1), \ldots, m\left(s_{0}\right)\right) \in \mathbb{N}^{s_{0}}$ and

$$
y \in \bigcup W_{m(1)} \cdots W_{m\left(s_{1}\right)},
$$

where the union is taken over all $\left(m(1), \ldots, m\left(s_{1}\right)\right) \in \mathbb{N}^{s_{1}}$ for some $s_{0}$ and $s_{1}$. Therefore,

$$
x \in W_{m^{\prime}(1)} \cdots W_{m^{\prime}\left(s_{0}\right)} \text { and } y \in W_{m^{\prime \prime}(1)} \cdots W_{m^{\prime \prime}\left(s_{1}\right)}
$$

for some $\left(m^{\prime}(1), \ldots, m^{\prime}\left(s_{0}\right)\right) \in \mathbb{N}^{s_{0}}$ and $\left(m^{\prime \prime}(1), \ldots, m^{\prime \prime}\left(s_{1}\right)\right) \in \mathbb{N}^{s_{1}}$. Thus,

$$
\begin{gathered}
x y \in W_{m^{\prime}(1)} \cdots W_{m^{\prime}\left(s_{0}\right)} W_{m^{\prime \prime}(1)} \cdots W_{m^{\prime \prime}\left(s_{1}\right)} \subset \\
\bigcup W_{m(1)} \cdots W_{m\left(s_{0}+s_{1}\right)} \subset W,
\end{gathered}
$$

where the union is taken over all $\left(m(1), \ldots, m\left(s_{0}+s_{1}\right)\right) \in \mathbb{N}^{s_{0}+s_{1}}$. By Lemma 3.1, the absolutely $k$-convex hull of any idempotent set is idempotent and $k$-convex. So,

$$
W^{\prime}:=\Gamma_{k}(W) \subset \Gamma_{k}\left(V_{1}\right)=V_{1} \subset U
$$

is an $m$ - $(k$-convex $)$ subset of $U$. Since

$$
W^{\prime} \cap E_{n}=\Gamma_{k}(W) \cap E_{n} \supset W \cap E_{n} \supset W_{n}=V_{n, n}^{\prime \prime}
$$

for each $n \in \mathbb{N}$ and $V_{n, n}^{\prime \prime}$ is an neighborhood of zero in $E_{n}$, then $W^{\prime}$ in $E$ is an absolutely $m$-( $k$-convex) neighborhood of zero.

Thus, $E$ is a commutative locally $m$-pseudoconvex algebra in the locally pseudoconvex inductive limit topology on $E$. 
A topological algebra is locally idempotent if it has a base of idempotent neighborhoods of zero (see [1, p. 196]). Hence, every locally $m$-pseudoconvex (in particular, locally $m$-convex) algebra is a locally idempotent algebra.

Theorem 4.2. Let $E$ be a topological inductive limit of a sequence of commutative locally idempotent algebras $E_{n}$ with continuous inclusions. If the multiplication is jointly continuous in $E$, then $E$ is a commutative locally idempotent algebra.

Proof. The proof is similar that of Theorem 2.

Acknowledgement. Research is in part supported by institutional research funding IUT20-57 of the Estonian Ministry of Education and Research, by the European Union through the European Social Fund (MOBILITAS grant No. MJD247) and by Consejo Nacional de Ciencia y Tecnología (CONACyT), Meéxico.

The authors would like to thank the referee for careful reading of the manuscript and for really useful remarks.

\section{REFERENCES}

1. M. Abel, Structure of locally idempotent algebras, Banach J. Math. Anal. 1 (2007), no. 2, 195-207.

2. M. Abel and R.M. Pérez-Tiscareño, Locally pseudoconvex inductive limit of topological algebras, Mediterr. J. Math. 4 (2013), no. 10, 1949-1963.

3. M. Akkar and C. Nacir, Structure m-convexe d'une algèbre limite inductive localement convexe d'algèbres de Banach, Rend. Sem. Mat. Univ. Padova 95 (1996), 107-126.

4. A. Arosio, Locally convex inductive limits of normed algebras, Rend. Sem. Mat. Univ. Padova 51 (1974), 333-359.

5. V.K. Balachandran, Topological Algebras, North-Holland Math. Studies 185, Elsevier, Amsterdam, 2000.

6. S. Dierolf and J. Wengenroth, Inductive limits of topological algebras, Linear Topol. Spaces Complex Anal. 3 (1997), 45-49.

7. T. Heintz and J. Wengenroth, Inductive limits of locally m-convex algebras, Bull. Belg. Math. Soc. Simon Stevin 11 (2004), no. 1, 149-152.

8. H. Hogbe-Nlend, Les fondements de la théorie spectrale des algèbres bornologiques, Bol. Soc. Brasi. Mat. 3 (1972), 19-56.

9. G. Köthe, Topological vector spaces I, Springer-Verlag, Berlin, Heiderberg, 1969.

10. A. Mallios, Topological Algebras. Selected Topics, North-Holland, Amsterdam, 1986.

11. E.A. Michael, Locally multiplicatively convex topological algebras, Mem. Amer. Math. Soc. 1952 (1952). no. 11, 79 pp.

12. M.P. Turpin, Sur une classe d'algebres topologiques generalisant les algebres localement bornees, Ph. D. Thesis, Faculty of sciences, University of Grenoble, 1966.

13. L. Waelbroeck, Topological vector spaces and algebras, Lecture Notes in Math. 230, SpringerVerlag, Berlin, New York, 1971.

14. S. Willard, General Topology, Addison-Wesley Publ. Co., Reading, Mass.-London-Don Mills, Ont., 1970.

1 Institute of Mathematics, University of Tartu, 2 J. Linvi Str., room 614, 50409 TARTU, Estonia.

E-mail address: mati.abel@ut.ee 
2 Departament of Mathematics, Autonomous Metropolitan University (UAM) Iztapalapa (Ixtapalapa) Campus, Av San Rafael Atlixco No.186 Col.Vicentina, Room AT-242, 09340 Mexico DF, Mexico.

E-mail address: reynapt@xanum.uam.mx 